Artigo original

\author{
Douglas Fernando Dias ${ }^{1}$ \\ Isabel Cristina Brisotti dos Reis ${ }^{1}$ \\ David Augusto dos Reis ${ }^{1}$ \\ Edilson Serpeloni Cyrino \\ David Ohara ${ }^{1}$ \\ Ferdinando Oliveira Carvalho ${ }^{1}$ \\ Juliano Casonatto ${ }^{1}$ \\ Mathias Roberto Loch ${ }^{1}$
}

\title{
COMPARAÇÃO DA APTIDÃO FÍSICA RELACIONADA À SAÚDE DE ADULTOS DE DIFERENTES FAIXAS ETÁRIAS
}

\author{
COMPARISON OF THE HEALTH RELATED PHYSICAL FITNESS OF ADULTS OF \\ DIFFERENTS AGES
}

\begin{abstract}
RESUMO
O objetivo deste estudo foi investigar as possíveis diferenças nos níveis de aptidão física relacionada à saúde (AFRS) em homens e mulheres de diferentes faixas etárias. Fizeram parte da amostra 326 sujeitos (132 homens e 194 mulheres), não-praticantes de exercícios físicos regulares, que foram separados, de acordo com o sexo, em três grupos etários: 20-29 anos (G1), 30-39 anos (G2) e 40-49 anos (G3). Todos os sujeitos foram submetidos a medidas antropométricas e de desempenho motor (flexibilidade, resistência/força muscular e aptidão cardiorrespiratória). A análise estatística incluiu o teste de Levene para verificação da homogeneidade das variâncias, estatística descritiva, ANOVA de um fator (faixa etária) e o teste de Bonferroni. O nível de significância adotado foi de $P<0,05$. Os melhores níveis de aptidão física, tanto nos homens quanto nas mulheres, foram observados nos sujeitos mais jovens (G1), enquanto que os piores níveis foram encontrados nos sujeitos de idade mais avançada (G3). A variação da gordura corporal relativa do G1 para o G3 foi mais acentuada nos homens (34\%) do que nas mulheres (16\%). Em relação ao desempenho motor, as maiores diferenças percentuais entre G1 e G3 foram identificadas no teste abdominal modificado (39\% vs. $22 \%$ para homens e mulheres, respectivamente) e flexão e extensão de braço (31\% vs. $24 \%$ para homens e mulheres, respectivamente). Os resultados sugerem que existe uma tendência natural de redução dos níveis de AFRS com o avançar da idade, em indivíduos não praticantes de exercícios físicos regulares.
\end{abstract}

Palavras-chave: Envelhecimento; Aptidão física; Saúde.

\section{ABSTRACT}

The objective of this study was to investigate possible differences in the levels of health related physical fitness of men and women of different ages. The sample comprised 326 people (132 men and 194 women) who did not perform regular physical exercise, and who were separated for analysis by sex and into three age groups: 20-29 years (G1), 30-39 years (G2) and 40-49 years (G3). All subjects underwent anthropometric measurements and motor performance testing (flexibility, muscle resistance/strength and cardiorespiratory fitness). Statistical analysis employed the Levene test to verify the homogeneity of variation, descriptive statistics, single factor ANOVA (age group) and the Bonferroni test. The significance level adopted was $p<0.05$. The best levels of physical fitness were observed among the youngest subjects (G1), irrespective of sex, while the worst fitness levels were detected in the oldest subjects (G3). The variation in body fat from G1 to G3 was more accentuated among the men (34\%) than among the women (16\%). With relation to motor performance, the greatest percentage differences between G1 and G3 were detected by either modified abdominal test (39\% and $22 \%$, for men and women respectively) and the arm flexion and extension test (31\% and $24 \%$, for men and women respectively. The results suggest that among individuals who do not regularly practice physical exercise there is a natural tendency for health related physical fitness to reduce as age increases.

Key words: Aging; Physical fitness; Health.

1 Grupo de Estudo e Pesquisa em Metabolismo, Nutrição e Exercício. Centro de Educação Física e Esportes. Universidade Estadual de Londrina. PR - Brasil. 


\section{INTRODUÇÃO}

A redução dos níveis atividade física habitual e aptidão física de indivíduos de ambos os sexos, em diferentes faixas etárias, é uma situação cada vez mais freqüente na maioria dos países do mundo. Esse fato se contrapõe àqueles observados em outros períodos históricos, nos quais a manutenção de bons níveis de aptidão física se fazia fundamental para a sobrevivência, haja vista que atividades como a caça, a pesca e a agricultura exigiam níveis consideráveis de aptidão física' ${ }^{1}$.

Por outro lado, paralelo a esta diminuição da exigência da aptidão física na vida cotidiana das pessoas, diversas evidências têm sido acumuladas a respeito dos benefícios da prática regular de atividade física e da manutenção de níveis adequados de aptidão física ${ }^{2-4}$.

Nesse sentido, os componentes da aptidão física que estão associados à redução dos níveis de morbi-mortalidade e/ou com um melhor desempenho nas atividades diárias são: aptidão cardiorrespiratória, resistência/força muscular, flexibilidade e composição corporal $^{1,5}$

Portanto, acredita-se que a manutenção de níveis adequados de aptidão física relacionada à saúde (AFRS) pode contribuir sobremaneira para o aumento da longevidade. Essa perspectiva tem atraído, sobretudo, a atenção da comunidade científica, bem como de órgãos governamentais, uma vez que o processo de envelhecimento, caracterizado por um decréscimo nas capacidades estruturais e funcionais do organismo, pode levar à diminuição da mobilidade e da competência funcional ${ }^{6-9}$.

Considerando que o avançar da idade, especialmente quando associado ao baixo nível de prática de atividade física, pode trazer conseqüências deletérias para a saúde e qualidade de vida da população, diversos pesquisadores têm procurado investigar a capacidade funcional e/ou o nível de aptidão física de idosos ${ }^{7,9}$, o que não tem sido um fato comum em adultos jovens e de meia-idade.

Além do mais, a maior parte dos estudos mencionados anteriormente, que comparam variáveis da aptidão física em sujeitos de diferentes faixas etárias, incluía apenas sujeitos praticantes de exercício físico.

Neste sentido, este estudo procurou estabelecer comparações entre o perfil de AFRS de adultos nãopraticantes de exercícios físicos, de ambos os sexos, em diferentes faixas etárias.

\section{PROCEDIMENTOS METODOLÓGICOS}

\section{Caracterização da amostra}

Para elaboração deste estudo foram analisados 326 sujeitos, sendo 132 homens e 194 mulheres, todos não-praticantes de exercícios físicos de maneira regular há pelo menos seis meses. Os indivíduos foram selecionados voluntariamente ao adentrarem em uma academia de ginástica e foram posteriormente divididos, de forma arbitrária em três grupos etários: 20 a 29 anos (G1), 30 a 39 anos (G2) e 40 a 49 anos
(G3). A tabela 1 apresenta a distribuição da amostra, de acordo com sexo e faixa etária.

Tabela 1. Distribuição da amostra segundo sexo e faixa etária.

\begin{tabular}{ccccc}
\hline & $\begin{array}{c}\text { G1 } \\
(20 \text { a } 29 \\
\text { anos })\end{array}$ & $\begin{array}{c}\text { G2 } \\
(30 \text { a 39 } \\
\text { anos })\end{array}$ & $\begin{array}{c}\text { G3 } \\
(40 \text { a } 49 \\
\text { anos })\end{array}$ & Total \\
\hline Masculino & 73 & 33 & 26 & 132 \\
Feminino & 123 & 39 & 32 & 194 \\
Total & 196 & 72 & 58 & 326 \\
\hline
\end{tabular}

Os sujeitos, após serem informados sobre os objetivos do estudo e procedimentos aos quais seriam submetidos, assinaram Termo de Consentimento Livre e Esclarecido. O Comitê de Ética em Pesquisa da Universidade Estadual de Londrina aprovou os procedimentos deste estudo, de acordo com as normas da resolução 196/96 do Conselho Nacional de Saúde para pesquisas em seres humanos.

\section{Procedimentos para a coleta dos dados}

Inicialmente os indivíduos foram submetidos a medidas de massa corporal, estatura, circunferências e espessuras de dobras cutâneas. Posteriormente, os participantes executaram uma bateria de testes motores, na seguinte ordem: sentar-e-alcançar (SA), abdominal modificado em um minuto (ABD), flexão e extensão dos braços (FEB) e teste ergométrico ${ }^{10} \mathrm{em}$ cicloergômetro.

\section{Antropometria}

A massa corporal (MC) foi mensurada em uma balança de plataforma digital, da marca Urano (modelo PS 180A), com precisão de $0,1 \mathrm{~kg}$, e a estatura em um estadiômetro de madeira com precisão de $0,1 \mathrm{~cm}$, de acordo com os procedimentos descritos por Gordon et al. ${ }^{11}$. O índice de massa corporal (IMC) foi determinado pelo quociente massa corporal/estatura ${ }^{2}$, sendo a MC expressa em quilogramas e a estatura em metros.

A gordura corporal relativa $(\% \mathrm{G})$ foi determinada pela técnica de espessura de dobras cutâneas. Três medidas foram obtidas em cada ponto anatômico, em seqüência rotacional, no hemicorpo direito, sendo registrado o valor mediano. Para tanto, foram aferidas as espessuras de dobras cutâneas abdominal, peitoral e coxa, nos homens, e suprailíaca, tricipital e coxa, nas mulheres. Tais medidas foram realizadas por um único avaliador, com um adipômetro da marca Cescorf, de acordo com as técnicas descritas por Slaughter et al. ${ }^{12}$. O coeficiente teste-reteste excedeu 0,95 para cada um dos pontos anatômicos com um erro de medida de no máximo $\pm 1,0 \mathrm{~mm}$. Para o cálculo da \%G, foi utilizada equação de $\mathrm{Siri}^{13}$, a partir da estimativa da densidade corporal obtida pelas equações propostas por Jackson \& Pollock ${ }^{14}$ para homens e Jackson et al. ${ }^{15}$ para mulheres.

Medidas de circunferências foram realizadas por meio de uma fita métrica metálica inextensível, com precisão de $0,1 \mathrm{~cm}$. A circunferência de cintura foi determinada no ponto médio entre a última costela $\mathrm{e}$ 
a crista ilíaca anterior, ao passo que, a circunferência de quadril foi medida na região de maior protuberância da região glútea, conforme as técnicas descritas por Callaway et al. ${ }^{16}$. As medidas foram feitas em duplicata pelo mesmo avaliador e o coeficiente teste-reteste excedeu 0,97 para cada um dos pontos anatômicos, com um erro de medida de no máximo $\pm 0,5 \mathrm{~cm}$.

\section{Testes Motores}

Como indicador de flexibilidade foi utilizado o teste de SA (AAHPERD) ${ }^{17}$. Cada indivíduo foi submetido a três tentativas, com intervalo de aproximadamente 30s entre elas, sendo registrado o maior valor obtido.

Como indicadores de resistência/força muscular, foram utilizados os testes de $\mathrm{ABD}^{18}$ e de FEB. Com relação ao teste $A B D$, foi registrado o número máximo de repetições executadas de forma correta durante um minuto, ao passo que no teste de FEB, foram adotados os procedimentos descritos por Pollock \& Wilmore ${ }^{19}$, sendo considerado o número de repetições completas, executadas em uma única tentativa.

O teste ergométrico submáximo de Astrand ${ }^{10}$ foi utilizado como indicador de resistência cardiorrespiratória. O teste foi realizado em cicloergômetro da marca Movement Biocycle Eletromagnetic.

\section{Tratamento estatístico}

Para verificar a homogeneidade das variâncias, utilizou-se o teste de Levene. Em função dessa primeira análise, optou-se por ANOVA de um fator (para a comparação entre as faixas etárias de acordo com o sexo). Quando detectadas diferenças significantes entre os grupos etários, o teste de Bonferroni foi adotado para a localização dessas diferenças. Em todos os procedimentos o nível de significância estabelecido foi de $P<0,05$. Os dados foram analisados no programa SPSS versão 11.5 .

\section{RESULTADOS}

As tabelas 2 e 3 apresentam a comparação dos resultados das variáveis analisadas, de acordo com o sexo. Foram observadas diferenças significantes, tanto em homens quanto em mulheres, com o avançar da idade. As variáveis IMC, relação cintura-quadril (RCQ) e \%G apresentaram valores crescentes com o avanço das faixas etárias, ao contrário do verificado no teste $\mathrm{ABD}$ e na variável $\mathrm{VO}_{2 \text { máx }}$. Por outro lado, não foram encontradas diferenças significantes com o avançar da idade no desempenho motor, nos testes motores SA e FEB, em indivíduos de ambos os sexos.

O teste de Bonferroni indicou que o G1 apresentou valores superiores ao $\mathrm{G} 2$ nas seguintes variáveis: $\mathrm{VO}_{2 \text { máx }}$ (em ambos os sexos), RCQ e \%G (somente nos homens) e ABD (somente nas mulheres). Por outro lado, diferenças estatisticamente significantes entre o G1 e o G3, em ambos os sexos, foram constatadas nas variáveis: IMC, RCQ e \%G (G1 < G3), ABD e VO ${ }_{2 \text { máx }}$

Tabela 2. Valores médios ( \pm DP) de variáveis morfológicas e de desempenho motor em homens adultos, em diferentes faixas etárias.

\begin{tabular}{|c|c|c|c|c|}
\hline & $\begin{array}{l}\mathrm{G} 1(\mathrm{n}=73) \\
20-29 \text { anos }\end{array}$ & $\begin{array}{l}\mathrm{G} 2(\mathrm{n}=33) \\
30-39 \text { anos }\end{array}$ & $\begin{array}{l}\mathrm{G} 3(\mathrm{n}=26) \\
40-49 \text { anos }\end{array}$ & $P$ \\
\hline IMC $\left(\mathrm{kg} / \mathrm{m}^{2}\right)$ & $25,4 \pm 4,2$ & $27,5 \pm 4,3^{a}$ & $27,6 \pm 4,7^{b}$ & 0,020 \\
\hline $\mathrm{RCQ}$ & $0,86 \pm 0,05$ & $0,90 \pm 0,05^{a}$ & $0,93 \pm 0,06^{b}$ & $<0,001$ \\
\hline$\% G$ & $19,5 \pm 8,4$ & $24,5 \pm 7,2^{\mathrm{a}}$ & $26,3 \pm 8,7^{b}$ & $<0,001$ \\
\hline $\mathrm{SA}(\mathrm{cm})$ & $19,8 \pm 8,9$ & $18,5 \pm 8,5$ & $17,9 \pm 8,4$ & 0,563 \\
\hline ABD (rep/min) & $33,3 \pm 9,1$ & $28,6 \pm 9,7$ & $20,2 \pm 9,7^{\mathrm{b}, \mathrm{c}}$ & $<0,001$ \\
\hline FEB (rep) & $18,9 \pm 10,1$ & $17,4 \pm 11,0$ & $14,7 \pm 8,2$ & 0,385 \\
\hline $\mathrm{VO}_{2 \max }(\mathrm{ml} / \mathrm{kg} / \mathrm{min})$ & $31,1 \pm 7,8$ & $26,9 \pm 5,4^{a}$ & $24,8 \pm 5,5^{b}$ & 0,001 \\
\hline
\end{tabular}

Nota. IMC = índice de massa corporal; $R C Q=$ relação cintura-quadril; \%G = gordura corporal relativa; $S A=$ teste sentar-

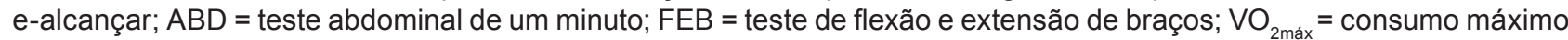
de oxigênio; rep = repetições. ${ }^{a} \mathrm{G} 1 \neq \mathrm{G} 2,{ }^{\mathrm{b}} \mathrm{G} 1 \neq \mathrm{G} 3 \mathrm{e}^{\mathrm{c}} \mathrm{G} 2 \neq \mathrm{G} 3$.

Tabela 3. Valores médios ( $\pm \mathrm{DP}$ ) de variáveis morfológicas e de desempenho motor em mulheres adultas, em diferentes faixas etárias.

\begin{tabular}{|c|c|c|c|c|}
\hline & $\begin{array}{l}\mathrm{G} 1(\mathrm{n}=123) \\
20-29 \text { anos }\end{array}$ & $\begin{array}{l}\mathrm{G} 2(\mathrm{n}=39) \\
30-39 \text { anos }\end{array}$ & $\begin{array}{l}\text { G3 }(n=32) \\
40-49 \text { anos }\end{array}$ & $P$ \\
\hline $\mathrm{IMC}\left(\mathrm{kg} / \mathrm{m}^{2}\right)$ & $23,3 \pm 3,8$ & $23,6 \pm 3,6$ & $25,8 \pm 3,5^{b, c}$ & 0,003 \\
\hline $\mathrm{RCQ}$ & $0,74 \pm 0,05$ & $0,76 \pm 0,05$ & $0,78 \pm 0,05^{b}$ & 0,009 \\
\hline$\% G$ & $30,1 \pm 7,0$ & $30,2 \pm 7,4$ & $34,9 \pm 7,4^{b, c}$ & 0,003 \\
\hline $\mathrm{SA}(\mathrm{cm})$ & $24,1 \pm 9,7$ & $22,5 \pm 8,5$ & $24,6 \pm 9,2$ & 0,574 \\
\hline ABD (rep/min) & $22,4 \pm 8,2$ & $18,5 \pm 8,9^{a}$ & $15,4 \pm 8,7^{b}$ & $<0,001$ \\
\hline $\mathrm{VO}_{2 \text { máx }}(\mathrm{ml} / \mathrm{kg} / \mathrm{min})$ & $26,0 \pm 5,1$ & $23,3 \pm 5,2^{a}$ & $21,8 \pm 4,5^{\mathrm{b}}$ & $<0,001$ \\
\hline
\end{tabular}

Nota. IMC = índice de massa corporal; $\mathrm{RCQ}=$ relação cintura-quadril; \%G = gordura corporal relativa; $\mathrm{SA}=$ teste sentare-alcançar; $\mathrm{ABD}=$ teste abdominal de um minuto; FEB = teste de flexão e extensão de braços; $\mathrm{VO}_{2 \text { máx }}=$ consumo máximo de oxigênio; rep = repetições. ${ }^{a} \mathrm{G} 1 \neq \mathrm{G} 2,{ }^{\mathrm{b}} \mathrm{G} 1 \neq \mathrm{G} 3 \mathrm{e}^{\mathrm{c}} \mathrm{G} 2 \neq \mathrm{G} 3$. 
(G1 > G3). Adicionalmente, diferenças significantes entre $G 2$ e G3 foram encontradas no ABD (G2 > G3, somente nos homens) e IMC e \%G (G2<G3, somente nas mulheres).

Considerando os aspectos morfológicos, verificou-se que a $\% \mathrm{G}$ foi a variável que apresentou maior diferença percentual quando comparados os grupos G1 e G3, sendo que a variação observada no sexo masculino foi muito mais elevada do que entre as mulheres $(34 \%$ e $16 \%$, respectivamente, para homens e mulheres).

Por outro lado, em relação ao desempenho motor, as maiores diferenças percentuais foram verificadas nos testes ABD e FEB, sendo registrados, respectivamente, os valores de $39 \%$ e $22 \%$, para o sexo masculino, e $31 \%$ e $24 \%$, para o sexo feminino.

\section{DISCUSSÃO}

Diferente de outros estudos que se propuseram a investigar os componentes da AFRS, sobretudo em crianças $^{20,21}$ e idosos ${ }^{22}$, o presente trabalho procurou, mediante corte transversal, investigar o perfil desses componentes em adultos jovens e de meia idade, de ambos os sexos.

Vale destacar que todos os participantes encontravam-se, quando da coleta dos dados, há pelo menos seis meses sem praticar exercícios físicos de maneira regular. $O$ controle dessa variável parece não ter sido uma das preocupações em outros estudos que procuraram, de maneira similar, investigar tal fenômeno. Acredita-se que no presente estudo a influência dessa variável tenha sido, pelo menos em parte, atenuada.

Outro aspecto a ser destacado é que a bateria de testes utilizada para avaliação dos componentes da aptidão física foi estruturada de modo que a capacidade física predominantemente exigida não fosse comprometida por esforços precedentes. Nesse sentido, os testes motores que exigem menor esforço foram realizados primeiro, evitando possíveis influências do desgaste físico acumulado nos testes subseqüentes (fadiga residual).

Na comparação entre os valores médios obtidos nos indicadores de composição corporal, observaramse diferenças crescentes entre os grupos no IMC, RCQ e \% gordura, indicando que o avanço da idade parece estar associado a um aumento na massa corporal total e da massa gorda em indivíduos não-treinados fisicamente.

Os achados do presente estudo corroboram os dados de Assis et al. ${ }^{23}$ que, ao investigarem 120 mulheres de 20 a 70 anos, participantes de um programa de atividade física orientada, verificaram que a diferença na $\% \mathrm{G}$ entre o grupo com idade entre 20 e 29 anos e o grupo com idade entre 40 e 49 anos era de aproximadamente $11 \%$, resultado bastante próximo aos $16 \%$ encontrados no presente trabalho.

Acredita-se que as diferenças entre os grupos encontradas neste estudo podem estar relacionadas, pelo menos em parte, à baixa oxidação de lipídios durante atividades do cotidiano, devido ao declínio paralelo no $\mathrm{VO}_{2 \max x}$. Existem indicativos de que a prática regular de atividades físicas possa reverter esta situação, tendo em vista que indivíduos treinados possuem maior estoque intracelular de lipídios e atividade da enzima lipoproteína lipase, a qual aumenta a oxidação de ácidos graxos ${ }^{24}$. Entretanto, não foram realizadas medidas bioquímicas para análise dessa hipótese neste estudo. Vale ressaltar que todos os grupos de ambos os sexos enquadraram-se na zona de risco moderado no que se refere à $R C Q$.

Embora alguns pesquisadores tenham indicado que o avançar da idade está associado a uma redução nos níveis de flexibilidade e força muscular ${ }^{26}$, o presente estudo não confirmou tal hipótese, tanto nos homens quanto nas mulheres, que foram analisadas por meio do desempenho motor nos testes de SA e FEB, respectivamente.

Por outro lado, um dos fatores que pode explicar, pelo menos em parte, as diferenças estatisticamente significantes observadas no teste motor de ABD em homens e mulheres nas diferentes faixas etárias, parece ser a perda de área transversa total que pode chegar a $10 \%$ entre 24 e 50 anos decorrente do processo de envelhecimento ${ }^{27}$. Spirduso ${ }^{28}$ destaca que os músculos de atividade especializada são os que sofrem maior declínio com o avançar da idade. Talvez, por esse motivo não tenham sido observadas diferenças significantes no teste de FEB (músculos que são exigidos constantemente), ao passo que, no teste $A B D$, diferenças foram identificadas (músculos com atividade mais especializada, e, portanto, menos exigidos) ao longo das diferentes faixas etárias.

Com relação ao $\mathrm{VO}_{2 \text { máx }}$ foram encontradas diferenças entre os grupos, em ambos os sexos, assumindo comportamento semelhante ao verificado no teste de $A B D$, no qual os valores médios do $G 3$ foram menores, sugerindo que indivíduos mais jovens possuem uma melhor condição cardiorrespiratória quando comparados aos de idade mais avançada.

Embora os resultados desse estudo com relação ao $\mathrm{VO}_{2 \text { máx }}$ corroborem as informações disponíveis na literatura, a magnitude dos achados obtidos em outras investigações que, de maneira similar, procuraram investigar o mesmo fenômeno, foi diferenciada.

Nesse sentido, Ravagnani et al. ${ }^{29}$, investigando 119 homens de 20 a 69 anos, encontraram diferenças de $32 \%$ do G1 (20-29) para o G3 (40-49) anos, ao passo que na presente investigação a diferença foi de aproximadamente $20 \%$. É importante salientar que as diferenças nas magnitudes entre as investigações podem ser atribuídas, pelo menos em parte, aos diferentes níveis de atividade física habitual, visto que os indivíduos do estudo supracitado estavam engajados em um programa de atividade física, ao passo que os participantes do presente estudo estavam a pelo menos seis meses sem praticar exercícios físicos de forma regular.

Entre as limitações do presente estudo está o uso do compasso (Cescorf), que não foi o mesmo usado no desenvolvimento das equações adotadas (Lange), podendo, assim, ter influenciado em parte os 
resultados e, conseqüentemente, a classificação dos sujeitos investigados. Entretanto, vale destacar que este foi um tipo de erro sistemático, não tendo grande influência na comparação entre as diferentes faixas etárias. Outro aspecto a ser destacado é que as coletas foram realizadas em diferentes condições (horário e temperatura diferenciadas).

Apesar dos sujeitos deste estudo terem referido, quando do momento da coleta das informações, não praticar exercícios físicos de maneira regular há pelo menos seis meses, não se pode afirmar que todos tinham o mesmo nível de atividade física habitual. Sugere-se que futuras investigações procurem controlar essa variável, bem como os hábitos nutricionais e o tabagismo, já que estas variáveis podem influenciar tanto a composição corporal quanto o desempenho dos sujeitos em testes motores. Outra sugestão é a inclusão, em futuros estudos dessa natureza, de sujeitos pertencentes a faixas etárias mais avançadas.

\section{CONCLUSÃO}

A partir dos resultados encontrados neste estudo, é possível concluir que os níveis de AFRS tendem a ser inferiores entre adultos não-treinados fisicamente com maior idade quando comparados a seus pares mais novos. Considerando que a manutenção de níveis adequados de AFRS se configura como um importante fator de proteção para uma série de disfunções crônicodegenerativas, além de estar associada a uma maior disposição para a realização de atividades diárias, reforça-se a importância da prática de exercícios físicos regulares, na tentativa de minimizar o impacto do envelhecimento sobre indicadores de saúde.

\section{REFERÊNCIAS BIBLIOGRÁFICAS}

1. Glaner MF. Importância da aptidão física relacionada à saúde. Rev Bras Cineantropom Desempenho Hum 2003;5(2):75-85.

2. Lee $C D$, Blair SN. Cardiorespiratory fitness and stroke mortality in men. Med Sci Sports Exerc 2002;34(4):5925.

3. Carnethon MR, Gidding SS, Nehgme R, Sidney S, Jacobs DR, Liu K. Cardiorespiratory fitness in young adulthood and the development of cardiovascular disease risk factors. JAMA 2003;290(23):3092-100.

4. Wessel TR, Arant CB, Olson MB, Johnson BD, Reis SE, Sharaf BL, et al. Relationship of physical fitness vc body mass index with coronary artery disease and cardiovascular events in women. JAMA 2004;292(10):1179-87.

5. Nahas MV. Atividade física, saúde e qualidade de vida. Londrina: Midiograf; 2003.

6. Matsudo SM, Matsudo VKR, Barros Neto TL, Araújo TL. Evolução do perfil neuromotor e capacidade funcional de mulheres fisicamente ativas de acordo com a idade cronológica. Rev Bras Med Esporte 2003;9(6):365-76.

7. Rosa TEC, Benício MHDA, Latorre MRDO, Ramos LR. Fatores determinantes da capacidade funcional entre idosos. Rev Saúde Pública 2003;37(1):40-8.

8. Raso V. A adiposidade corporal e a idade prejudicam a capacidade funcional para realizar as atividades da vida diária de mulheres acima de 47 anos. Rev Bras Med Esporte. 2003;8(6):225-34.

9. Virtuoso Junior JS. Atividade física habitual e autonomia funcional de idosos em Florianópolis, SC. Dissertação de Mestrado em Educação Física, Universidade Federal de Santa Catarina. Florianópolis:SC; 2004.

10. Astrand PO, Rodahl K. Textbook of work physiology. New York: Mc Graw Hill; 1986.

11. Gordon CC, Chumlea WC, Roche AF. Stature, recumbent length, and weight. In: Lohman TG, Roche AF, Martorell $\mathrm{R}$, editors. Anthropometric standardization reference manual. Champaign: Human Kinetics Books; 1988:3-8.

12. Slaughter MH, Lohman TG, Boileau RA, Stillman RJ, Van Loan M, Horswill CA, et al. Influence of maturation on relationship of skinfolds to body density: a crosssectional study. Am J Hum Biol 1984;56(4):681-9.

13. Siri, WE. Body composition from fluid spaces and density: analysis of methods. In: Brozek J, Henschel A. editors. Techniques for measuring body composition. Washington: National Academy of Science; 1961:223-44.

14. Jackson AS, Pollock ML. Generalized equations for predicting body density of men. Br J Nutr 1978;40(3):497504.

15. Jackson AS, Pollock ML, Ward A. Generalized equations for predicting body density of women. Med Sci Sports Exerc 1980;12(3):175-81.

16. Callaway CW, Chumlea WC, Bouchard CR, Himes JH, Lohman TG, Martin AD, et al. Circumferences In: Lohman TG, Roche AF, Martorell R, editors. Anthropometric standardization reference manual. Champaign: Human Kinetics Books; 1988. p. 39-54.

17. The American Alliance for Health, Physical Education, Recreation and Dance. Health Related Physical Fitness Technical Manual, 1984.

18. Soares J, Sessa M. Medidas da força muscular. In: Matsudo VKR, editor. Testes em ciência do esporte. São Caetano do Sul: Gráfica Burti; 1987:57-68.

19. Pollock ML, Wilmore JH. Exercício na saúde e na doença: avaliação e prescrição para prevenção e reabilitação. Rio de Janeiro: Medsi; 1993.

20. Welk GJ, Schaben JA, Shelley M. Physical activity and physical fitness in children schooled at home and children attending public schools. Ped Exerc Sci 2004;16:310-23.

21. Bergman GG, Araújo MLB, Garlipp DC, Lorenzi TDC, Gaya A. Alteração anual no crescimento e na aptidão física relacionada à saúde de escolares. Rev Bras Cineantropom Desempenho Hum 2005;7(2):55-61.

22. Zago AS, Gobbi S. Valores normativos da aptidão funcional de mulheres de 60 a 70 anos. Rev Bras Ciênc Mov 2003;11(2):77-86.

23. Assis CR, Mesa AJR, Nunes VGS. Determinação da composição corporal de pessoas de 20 a 70 anos, da comunidade pelotense. Rev Bras Ciênc Mov 1999;1(1):82-8.

24. Hawley JA, Brouns F, Jeukendrup J. Strategies to enhance fat utilization during exercise. Sports Med 1998;25(4):241-57.

25. Lohman, TG. Advances in body composition assessment. Champaign: Human Kinetics Books; 1992.

26. Sato T, Demura S, Murase T, Kobayayashi $Y$. Contribution of physical fitness component to health status in middle-aged and elderly males. J Physiol Anthropol 2006;25(5):311-9.

27. Booth FW, Weeden SH, Tseng BS. Effect of aging on human skeletal muscle and motor function. Med Sci Sports Exerc. 1994;26:556-60. 
28. Spirduso W. Physical dimensions of aging. Champaign: Human Kinetics Books; 1995.

29. Ravagnani FCP, Coelho CF, Burini RC. Declínio do consumo máximo de oxigênio em função da idade em indivíduos adultos do sexo masculino submetidos ao teste ergoespirométrico. Rev Bras Ciênc Mov 2005;13(2):7-15.
AGRADECIMENTOS

Os autores agradecem pelo apoio financeiro da CAPES e CNPq pelas bolsas outorgadas.

\section{Endereço para correspodência:}

Ferdinando Oliveira Carvalho

Recebido em 16/07/07

Rodovia Celso Garcia Cid, Km 380.

Revisado em 20/08/07

Campus Universitário.

Aprovado em 15/09/07

CEP 86051-990 - Londrina, PR. Brasil.

E-mail: ferdinandocarvalho@hotmail.com 